DOI 10. 18307/2017. 0506

(c) 2017 by Journal of Lake Sciences

\title{
分级进水对阶梯垂直流人工湿地污水处理效果的影响”
}

\author{
沈林亚, 吴 娟, 钟 非, 向东方, 成水平** \\ (同济大学长江水环境教育部重点实验室,上海 200092)
}

\begin{abstract}
摘 要: 本文研究了分级进水对阶梯垂直流人工湿地处理生活污水效果的影响. 在保持总水力负荷为 $100 \mathrm{~mm} / \mathrm{d}$ 的情况 下, 分别将总进水量的 $0 \% 、 10 \% 、 20 \%$ 和 30\% 用于湿地第 2 级阶梯的进水, 分析了系统对化学需氧量 ( COD) 和氮、磷的总 去除率和沿程变化. 结果表明, 不同分级进水比例条件下人工湿地对 COD 和总磷 (TP) 的去除率差异不明显, COD 和 TP 的最高去除率分别为 $87.80 \%$ 和 $81.17 \%$, COD 的去除主要集中在第 1 级, 其贡献率平均为 $82.18 \%$, TP 的去除主要集中在 第 3 级, 贡献率平均为 $54.37 \%$. 分级进水对总氮 (TN) 去除率影响显著 $(P<0.05)$, 当 2 级进水比例为 $20 \%$ 时, $\mathrm{TN}$ 去除率最 高, 为 $61.70 \% \pm 4.48 \%$, 且 3 个梯级对 TN 的去除贡献率分别为 $36.52 \% 、 42.11 \%$ 和 $21.37 \%$. 相同组合形式的垂直流人工湿 地处理生活污水的工程应用中, 可根据需求设置 $20 \%$ 左右的 2 级进水比例, 以提高人工湿地对污染物的去除效果.
\end{abstract}

关键词: 阶梯垂直流人工湿地; 分级进水;脱氮除磷

\section{Effect of step feeding on the performance of multi-stage vertical flow constructed wetland for municipal wastewater treatment}

\author{
SHEN Linya, WU Juan, ZHONG Fei, XIANG Dongfang \& CHENG Shuiping ** \\ (Key Laboratory of Yangtze River Water Environment, Ministry of Education, Tongji University, Shanghai 200092, P.R.Chi- \\ na)
}

\begin{abstract}
To investigate the effect of step feeding on the performance of multi-stage vertical flow constructed wetland (MS-VFCW) for municipal wastewater treatment, raw wastewater with four step feeding ratios $(0 \%, 10 \%, 20 \%$ and $30 \%)$ were dosed to the second stage of the MS-VFCW plots at a total hydraulic load of $100 \mathrm{~mm} / \mathrm{d}$. The results showed no significant differences in removal of chemical oxygen demand ( COD) and total phosphorus (TP) among the four step feeding ratios, and the highest removal rates of COD and TP reached up to $87.80 \%$ and $81.17 \%$, respectively. Most COD was removed in the first stage, with an average contribution of $82.18 \%$. While the most TP was removed in the third stage, with an average contribution of $54.37 \%$. In contrast, the differences in total nitrogen ( TN) removal rates among the four step feeding ratios were significant, with the highest TN removal rates $(61.70 \% \pm 4.48 \%$ ) under the ratio of $20 \%$, in which the average removal contribution of each stage was $36.52 \%, 42.11 \%$ and $21.37 \%$, respectively. The step feeding ratio was recommended as $20 \%$ in application of the hybrid VFCWs so as to achieve sound pollutant removal performance.
\end{abstract}

Keywords: Multi-stage vertical flow constructed wetland; step feeding; removal of nitrogen and phosphorus

人工湿地能有效净化污染水体, 被广泛应用于点源和面源污染的治理. 垂直流人工湿地系统氧转移能 力较强 ${ }^{[1-2]}$, 硝化作用明显 ${ }^{[3]}$, 但反硝化作用往往受到限制, 而且碳源不足也是制约反硝化过程的重要因素 之一, 因此碳源补充是提高湿地系统反硝化能力、增强脱氮效率的有效途径之一. Zhang 等 ${ }^{[4]}$ 使用植物发酵 液作为人工湿地的反硝化碳源, 发现随着植物发酵液投加量的增加, 系统中硝酸盐的去除率显著增加. Sikora 等 ${ }^{[5]}$ 以低分子碳水化合物乙酸为外加碳源, 系统中硝酸盐的去除效率最高可达 $70 \%$, 可见低分子碳水 化合物可有效促进人工湿地的反硝化进程. 同时, 因为单一湿地类型不能同时提供充分的好氧和厌氧条件,

* 国家自然科学基金项目 (51578395) 和上海市创新行动计划项目 (16DZ1204803) 联合资助. $2017-04-18$ 收稿; 2017 - 05 - 27 收修改稿. 沈林亚 (1993 ), 女, 硕士研究生;E-mail:lyshen2015@ tongji.edu.cn.

** 通信作者; E-mail:shpcheng@ tongji.edu.cn. 
限制了污染物的去除效率 ${ }^{[6-7]}$, 人工湿地应用已不再局限于单一的水流方式, 研究者们开始将不同类型的人 工湿地进行组合 ${ }^{[8]}$. 本研究提出了一种阶梯垂直流人工湿地工艺, 采用下行流一上行流一下行流的组合形 式,强化了污染物的去除效果 ${ }^{[9]}$.

为了提高阶梯垂直流人工湿地的氮去除效率, 本研究将分级进水应用于阶梯垂直流人工湿地, 一方面 可以适应不同地形地势, 用以维持水体在系统内部的重力流形式; 另一方面通过分级进水为系统内反硝化 过程提供碳源, 加强脱氮效果. 本文通过分析比较不同进水配比下污染物的去除效果, 从而得出最优的进水 方式, 为阶梯组合式垂直流人工湿地的设计和运行提供合理参数.

\section{1 材料与方法}

\section{1 阶梯垂直流人工湿地小试系统}

共构建 4 套阶梯垂直流人工湿地小试系统. 每套人工湿地系统由 3 级垂直流人工湿地单元组合而成 (图 1), 分别填充 $0.45 \mathrm{~m}$ 高度的沸石、页岩和陶粒. 3 种填料的基本物理化学性质见表 1 . 湿地的 3 级单元均 种植西伯利亚感尾 ( Iris sibirica), 种植密度为 20 株 $/ \mathrm{m}^{2}$.

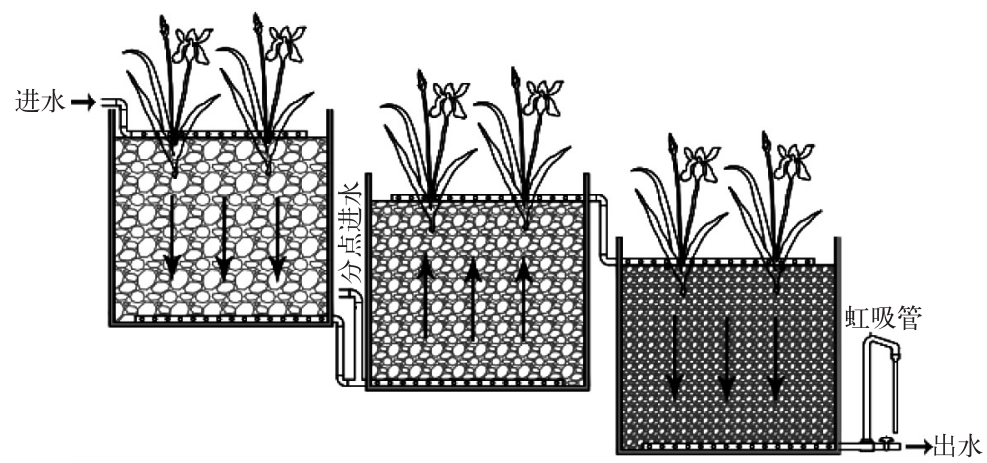

图 1 阶梯垂直流人工湿地小试系统

Fig.1 Lab-scale multi-stage vertical flow constructed wetland

每级湿地顶部和底部均设有直径为 $20 \mathrm{~mm}$ 的穿孔 PVC 管用于布水或集水. 第 3 级湿地出水口设置虹 吸管,虹吸管出口位于 $25 \mathrm{~cm}$ 处.

表 1 填料物理化学性质

Tab.1 Physical and chemical properties of the medias

\begin{tabular}{ccccc}
\hline 基质 & 粒径 $/ \mathrm{mm}$ & $\mathrm{pH}$ & 堆积密度 $/\left(\mathrm{g} / \mathrm{cm}^{3}\right)$ & 孔隙率 \\
\hline 沸石 & $8 \sim 16$ & 7.17 & 0.9968 & 0.58 \\
页岩 & $5 \sim 8$ & 8.57 & 0.7597 & 0.45 \\
陶粒 & $4 \sim 8$ & 10.87 & 0.9767 & 0.42 \\
\hline
\end{tabular}

\section{2 实验设计}

实验前用自来水冲洗湿地填料, 去除其表面灰尘和其他污染物. 取上海市杨浦区某污水厂初沉池出水 作为湿地进水, 进水化学需氧量 $(\mathrm{COD})$ 、总氮 $(\mathrm{TN})$ 、铵态氮 $\left(\mathrm{NH}_{4}^{+}-\mathrm{N}\right)$ 、硝态氮 $\left(\mathrm{NO}_{3}^{-}-\mathrm{N}\right)$ 、亚硝态氮 $\left(\mathrm{NO}_{2}^{-}-\mathrm{N}\right)$ 、 总磷 $(\mathrm{TP})$ 和磷酸盐 $\left(\mathrm{PO}_{4}^{3-}\right)$ 的浓度分别为 $142.3 \pm 24.5 、 36.83 \pm 3.81 、 32.68 \pm 1.93 、 0.87 \pm 0.18 、 0.05 \pm 0.12 、 4.39 \pm$ 0.92 和 $3.10 \pm 0.90 \mathrm{mg} / \mathrm{L}$. 对湿地系统微生物进行培养驯化, 水力负荷为 $60 \mathrm{~mm} / \mathrm{d}$, 定期测试出水水质, 直至处 理效果稳定后再提高进水负荷. 实验周期为 2016 年 4-8 月,历时 4 个月左右.

4 套系统总进水负荷均为 $100 \mathrm{~mm} / \mathrm{d}$, 设置 $0 \% 、 10 \% 、 20 \%$ 和 30\% 4 个不同的 2 级进水份额的处理方式: $0 \%$ 的第 1 级进水份额为 $100 \% ; 10 \%$ 的第 1 级进水份额为 $90 \%$, 第 2 级为 $10 \% ; 20 \%$ 的第 1 级进水份额为 
$80 \%$,第 2 级为 $20 \% ; 30 \%$ 的第 1 级进水份额为 $70 \%$,第 2 级为 $30 \%$.

\section{3 样品采集与测试}

实验期间采集每套湿地的第 $1 、 2$ 和 3 级出水和进水,用于水质分析, 采样频率为 1 次/周. 水质测试指标 为 $\mathrm{COD} 、 \mathrm{NH}_{4}^{+}-\mathrm{N} 、 \mathrm{NO}_{3}^{-}-\mathrm{N} 、 \mathrm{NO}_{2}^{-}-\mathrm{N} 、 \mathrm{TN} 、 \mathrm{PO}_{4}^{3-}$ 和 TP 浓度. $\mathrm{COD}$ 浓度采用哈希消解仪 DRB 200 和分光光度计 DR 2800 测定,其他指标均采用标准方法测定 ${ }^{[10]}$.

\section{4 数据处理}

污染物的去除率 $(\%)$ 计算公式为:

$$
\text { 去除率 }=\left(1-C_{\text {out }} / C_{\text {in }}\right) \times 100 \%
$$

污染物质量去除速率 $\left(\mathrm{g} /\left(\mathrm{m}^{2} \cdot \mathrm{d}\right)\right)$ 计算公式为:

$$
\text { 质量去除速率 }=\left(C_{\text {in }} \cdot V_{\text {in }}-C_{\text {out }} \cdot V_{\text {out }}\right) /(S \cdot H R T)
$$

式中, $C_{\text {in }}$ 和 $C_{\text {out }}$ 分别为进、出水浓度 $(\mathrm{mg} / \mathrm{L}) ; V_{\text {in }}$ 和 $V_{\text {out }}$ 分别为进、出水体积 $\left(\mathrm{m}^{3}\right) ; S$ 为人工湿地面积 $\left(\mathrm{m}^{2}\right)$; $H R T$ 为水力停留时间 $(\mathrm{d})$.

去除贡献率 $(\%)$ 计算公式为:

$$
\begin{aligned}
& \text { 系统第 } 1 \text { 级去除贡献率 }=\left(1 \text { 级 } C_{\text {in }}-1 \text { 级 } C_{\text {out }}\right) /\left(1 \text { 级 } C_{\text {in }}-3 \text { 级 } C_{\text {out }}\right) \times 100 \% \\
& \text { 系统第 } 2 \text { 级去除贡献率 }=\left(1 \text { 级 } C_{\text {out }}-2 \text { 级 } C_{\text {out }}\right) /\left(1 \text { 级 } C_{\text {in }}-3 \text { 级 } C_{\text {out }}\right) \times 100 \% \\
& \text { 系统第 } 3 \text { 级去除贡献率 }=\left(2 \text { 级 } C_{\text {out }}-3 \text { 级 } C_{\text {out }}\right) /\left(1 \text { 级 } C_{\text {in }}-3 \text { 级 } C_{\text {out }}\right) \times 100 \%
\end{aligned}
$$

使用 IBM SPSS 20 软件对数据进行统计分析, 采用单因素方差分析法分析不同分级进水比例条件下处 理效果的差异性, $P<0.05$ 表明具有显著性差异.

\section{2 结果与讨论}

\subsection{COD 去除效果}

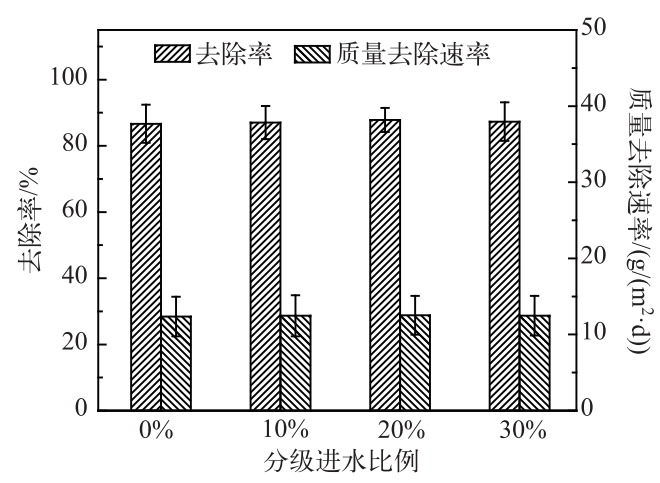

图 2 不同分级进水比例条件下阶梯 垂直流人工湿地对 COD 的去除效果

Fig.2 COD removal efficiencies of MS-VFCW under different step feeding ratios
4 种分级进水条件下人工湿地对 COD 的去除效果 差异不显著 (图 2), 其去除率为 $86.66 \% \sim 87.80 \%$, 质量 去除速率为 $12.36 \sim 12.53 \mathrm{~g} /\left(\mathrm{m}^{2} \cdot \mathrm{d}\right)$, 说明阶梯垂直流 人工湿地能有效适应本实验中 4 种分级进水方式, 普遍 具有较好的 COD 去除效果. 湿地系统的有机物主要通 过附着于基质上的生物膜和微生物的代谢去除 ${ }^{[11]}$, 垂 直流人工湿地系统充氧能力较好, 为微生物分解污染 物提供了有利条件. Vymazal 等 ${ }^{[3]}$ 研究不同进水方式下 复合垂直流人工湿地对 COD 的去除效果差异, 发现 COD 的去除效率均能稳定达到 $80 \%$, 且当进水负荷增 大时, COD 的去除效果也没有显著变化.

4 个分级进水条件下湿地系统进水和各级出水 COD 浓度如图 3 所示. 在进水浓度为 $142.3 \pm 24.5 \mathrm{mg} / \mathrm{L}$ 时, 4 套系统 3 级出水 COD 浓度平均值为 $17.5 \mathrm{mg} / \mathrm{L}$, 处理组间差异不显著. 出水 $\mathrm{COD}$ 浓度远低于《城镇污

水处理厂污染物排放标准》 (GB 18918-2002) 的一级 A 标准. 4 套系统的第 1 级对 COD 去除的贡献率平均 为 $82.18 \%$, 进水中大部分有机物在第 1 级得以去除, 这是因为污水从位于填料上部的布水管均匀流人, 与空 气接触后进人第 1 级,系统的好氧状况为 COD 的去除提供了有利条件. 4 套系统第 2 级对 COD 去除的平均 贡献率仅为 $1.29 \%$. 研究表明, 厌氧或缺氧条件下, 有机物可被厌氧自养细菌降解去除 ${ }^{[12]}$, 但其新陈代谢速 率远小于异养微生物, 对有机物的降解作用也较弱 ${ }^{[13]}$. 系统第 3 级出水 COD 浓度较第 2 级有明显降低, 对 COD 去除的贡献率平均为 $16.53 \%$. 因此本实验中 COD 的去除过程主要发生在湿地系统的第 1 级. 李剑 波 ${ }^{[14]}$ 研究了组合人工湿地对 COD 的去除效果, 并计算得到第 1 级垂直流人工湿地对 COD 去除的贡献率高 达 $92.6 \%$,垂直流人工湿地 COD 去除主要集中在第 1 级. 


\section{2 氮去除效果}

4 个进水条件下 TN 去除效果较为稳定，去除率分别为 $45.03 \% \pm 7.74 \% 、 48.74 \% \pm 6.05 \% 、 61.70 \% \pm 4.48 \%$ 和 $46.98 \% \pm$ $10.34 \%$, 对应的质量去除速率分别为 $1.67 \pm 0.38 、 1.81 \pm 0.39$ 、 $2.28 \pm 0.35$ 和 $1.75 \pm 0.53 \mathrm{~g} /\left(\mathrm{m}^{2} \cdot \mathrm{d}\right)$ (图 4). 分级进水比例为 $20 \%$ 时 TN 去除效果最好,去除率和质量去除速率明显高于 其他 3 种进水方式 $(P<0.05)$, 这与湿地中氮的去除机制有 关. $\mathrm{Li}$ 等 $^{[15]}$ 的研究发现, 采用分级进水方式后垂直流人工湿 地对 TN 的去除率可达 $60.6 \%$. 人工湿地中通过植物吸收和 填料吸附去除的氮含量仅占总去除量的 3\% 左右, 微生物降 解是氮素去除的最主要路径 ${ }^{[14]}$.

4 个进水条件下进水和各级出水中 $\mathrm{TN}$ 以 $\mathrm{NH}_{4}^{+}-\mathrm{N}$ 为主 (图 5). 进水 $\mathrm{TN}$ 浓度为 $36.83 \pm 3.81 \mathrm{mg} / \mathrm{L}, 4$ 套系统出水 $\mathrm{TN}$ 浓度分别为 $21.04 \pm 2.62 、 18.73 \pm 1.62 、 14.04 \pm 1.65$ 以及 $19.30 \pm$ $3.10 \mathrm{mg} / \mathrm{L}$. 出水 $\mathrm{TN}$ 浓度基本达到《城镇污水处理厂污染物 排放标准》( GB 18918-2002) 的一级 B 或者一级 A 标准. 从 湿地沿程变化来看, 每级出水 $\mathrm{TN}$ 浓度随梯级不断下降, 其 中分级进水比例为 $20 \%$ 的条件下第 1 级出水浓度为 27.66 $\mathrm{mg} / \mathrm{L}$, 较进水下降了 $9.17 \mathrm{mg} / \mathrm{L} .4$ 套系统第 1 级对 $\mathrm{TN}$ 去除 的贡献率分别为 $34.50 \% 、 41.51 \% 、 36.52 \%$ 和 $47.24 \%$. 考虑到 本实验系统第 1 级虽然是下向流,但填料底部也会存在庆氧 区域,一部分 $\mathrm{NO}_{3}^{-}-\mathrm{N}$ 被还原成 $\mathrm{N}_{2} \mathrm{O} 、 \mathrm{NO}$ 和 $\mathrm{N}_{2}$ 去除. 本研究 试验周期为 4-8 月, 温度较高, 且 4 套系统 1 级出水平均 $\mathrm{pH}$ 值为 7.63 , 具备厌氧氨氧化发生的条件, 研究表明, 在没 有任何接种和富集的情况下, 人工湿地系统中也有厌氧氨 氧化细菌存在, 可以 $\mathrm{NH}_{4}^{+}-\mathrm{N}$ 和 $\mathrm{NO}_{2}^{-}-\mathrm{N}$ 为电子供体和电子受 体, 将氮素转化成 $\mathrm{N}_{2}$ 排出系统 ${ }^{[16]}$. 此外, 阶梯垂直流人工湿 地第 1 级填充了对于 $\mathrm{NH}_{4}^{+}-\mathrm{N}$ 具有较好吸附作用的沸石, 部 分 $\mathrm{NH}_{4}^{+}-\mathrm{N}$ 可通过沸石的离子交换作用去除.

垂直流人工湿地为硝化作用提供了有利条件, 但反硝 化过程往往受到限制. 本研究采用从阶梯垂直流湿地第 2 级补充污水提供碳源, 结合上行水流为系统提供的戻氧环 境, 强化了反硝化作用, 提升了氮的去除效果, 因此系统第 2 级出水 $\mathrm{TN}$ 浓度也在第 1 级的基础上有大幅降低, 系统第 2 级对 TN 去除的贡献率分别为 $42.81 \% 、 40.56 \% 、 36.71 \%$ 和 $31.64 \%$. 但分级进水比例并不是越高越好, 比较不同系统 的 2 级出水氮元素组成, 发现随着 2 级进水比例的提高, 新 加人的 $\mathrm{NH}_{4}^{+}-\mathrm{N}$ 未能及时转化成 $\mathrm{NO}_{3}^{-}-\mathrm{N}$, 导致出水 $\mathrm{NH}_{4}^{+}-\mathrm{N}$ 浓 度有所增加. 二级进水导致出水 $\mathrm{NO}_{3}^{-}-\mathrm{N}$ 浓度明显下降, 分 级进水补充的碳源作为反硝化电子供体, 促进了 $\mathrm{NO}_{3}^{-}-\mathrm{N}$ 向 $\mathrm{N}_{2} \mathrm{O} 、 \mathrm{NO}$ 和 $\mathrm{N}_{2}$ 的转化.

比较各系统第 3 级 TN 出水浓度, 发现分级进水比例为 $20 \%$ 的第 3 级出水 TN 浓度显著低于其他 3 个处理组, 达到 了最高的 TN 去除效果, 3 个梯级对 TN 的去除贡献率分别

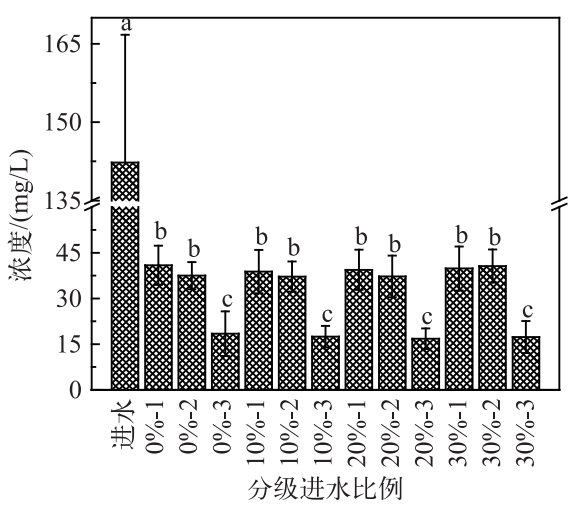

图 3 不同分级进水比例条件下阶梯 垂直流人工湿地中 COD 的沿程变化 $(1 、 2$ 和 3 表示湿地的第 $1 、 2$ 和 3 梯级, 不同字母表示差异显著,下同)

Fig.3 COD changes among stages of MS-VFCW under different step feeding ratios

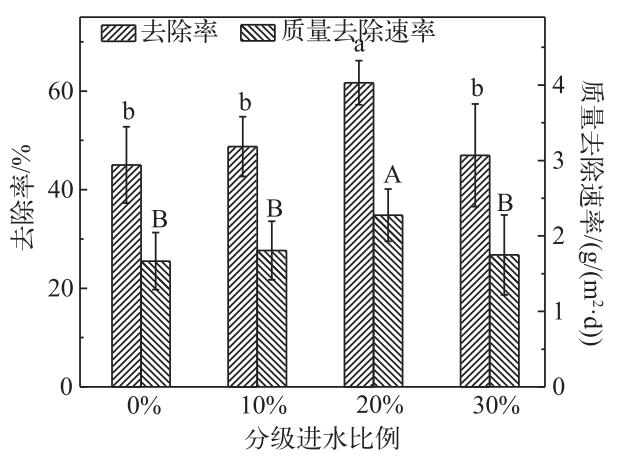

图 4 不同分级进水比例条件下阶梯 垂直流人工湿地对总氮的去除效果

Fig.4 Total nitrogen removal efficiencies of MS-VFCW under different step feeding ratios

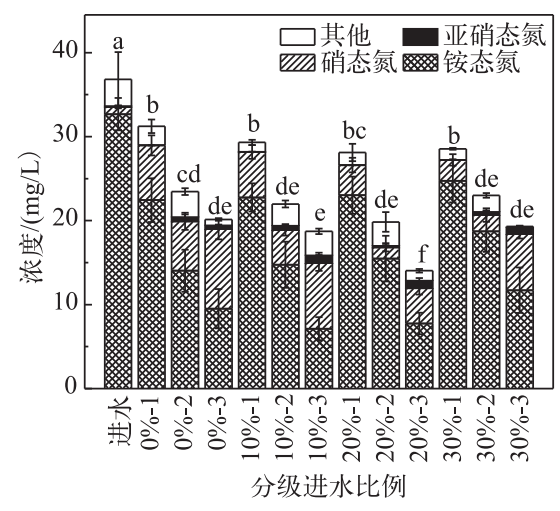

图 5 不同分级进水比例条件下阶梯 垂直流人工湿地中氮元素的沿程变化

Fig.5 Nitrogen changes among stages of MS-VFCW under different step feeding ratios 
为 $36.52 \% 、 42.11 \%$ 和 $21.37 \%$. 当第 2 级进水比例为 $30 \%$ 时, $\mathrm{TN}$ 的去除效率反而呈现下降趋势, 可能是因为 第 2 级补充的过多污水中的氮无法完成硝化过程, 导致整体的 TN 去除效率下降. 可见分级进水的比例必须 控制在合适范围内才能提升该湿地系统的除氮效果. Fan 等 ${ }^{[17]}$ 以蔗糖为外加碳源, 研究其不同添加量对垂 直流人工湿地 TN 去除效果的影响, 发现当碳氮比为 10 并添加曝气装置的情况下, TN 的去除效率可高达 $90 \%$. 本小试实验出水中仍有部分 $\mathrm{NH}_{4}^{+}-\mathrm{N}$ 剩余, 可考虑进水曝气以达到更高的 $\mathrm{TN}$ 去除效果. Zhao 等 ${ }^{[18]}$ 模 拟生活污水污染物的浓度进行人工配水, 研究不同碳氮比 $(\mathrm{C} / \mathrm{N})$ 条件下, 小型垂直流人工湿地脱氮效果的 差异性, 发现当 $\mathrm{C} / \mathrm{N}$ 在 2.5 5 时, $\mathrm{TN}$ 去除率普遍较高. 本研究以生活原污水为补充碳源, 随着分级进水比例 的提高, 2 级进水 $\mathrm{C} / \mathrm{N}\left(\mathrm{TOC} / \mathrm{NO}_{3}^{-}-\mathrm{N}\right)$ 由 1.83 上升到 3.06、5.50 和 10.14. 且当 $\mathrm{C} / \mathrm{N}$ 为 5.50 时, $\mathrm{TN}$ 去除率高达 $61.70 \%$,随着 $\mathrm{C} / \mathrm{N}$ 的进一步增加, TN 去除效率不升反降, 与 Zhao 等的结果类似.

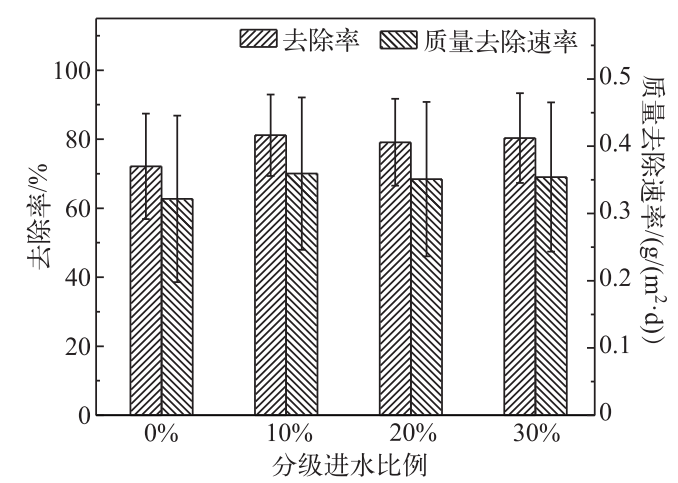

图 6 不同分级进水比例条件下阶梯 垂直流人工湿地对总磷的去除效果

Fig.6 Total phosphorus removal efficiencies of MS-VFCW under different step feeding ratios

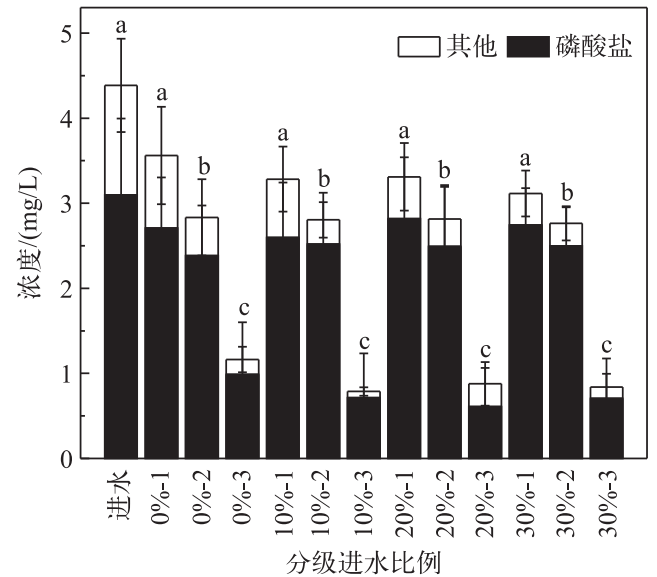

图 7 不同分级进水比例条件下阶梯 垂直流人工湿地中磷元素的沿程变化

Fig.7 Phosphorus changes among stages of MS-VFCW under different step feeding ratios

\section{3 磷去除效果}

4 个分级进水比例条件下, TP 去除率为 $72.14 \%$ $81.17 \%$, 质量去除速率为 $0.32 \sim 0.36 \mathrm{~g} /\left(\mathrm{m}^{2} \cdot \mathrm{d}\right.$ ) (图 6). 同 COD 相似,各处理间 TP 去除效果不存在显著性 差异, 说明进水方式对磷的去除影响不大. 实际上, 人 工湿地中主要通过填料吸附来实现磷的去 ${ }^{\text {除 }}{ }^{[19-20]}$. 本 实验出水 $\mathrm{TP}$ 浓度基本达到《城镇污水处理厂污染物排 放标准》( GB 18918-2002) 的一级 B 标准.

4 套系统进水和各级出水中的磷主要以 $\mathrm{PO}_{4}^{3-}$ 形式 存在,约占进水 TP 浓度的 $70.62 \% .4$ 个系统出水 TP 浓 度分别为 $1.16 \pm 0.66 、 0.79 \pm 0.52 、 0.87 \pm 0.54$ 和 $0.84 \pm$ $0.59 \mathrm{mg} / \mathrm{L}$, 各处理之间不存在显著差异 (图 7).

污水经湿地第 1 和第 2 级处理后 TP 浓度并未显 著降低, 但经第 3 级后其浓度明显减少, 4 套系统第 1 、 2 级对 TP 去除的贡献率平均分别为 $30.66 \%$ 和 $14.97 \%$, 第 3 级对 TP 去除的贡献率平均为 $54.37 \%$, 表明该湿 地系统对磷的去除主要在第 3 级完成. 第 3 级填料陶粒 中富含的氧化钙在基质与磷溶液的混合体系中, 与水分 子结合形成氢氧化钙, 进而电离出钙离子, 溶液中的磷 酸根离子最终通过非晶体的磷酸钙沉淀去除 ${ }^{[21-22]}$. 我们 在填料对 $\mathrm{KH}_{2} \mathrm{PO}_{4}$ 溶液等温吸附的前期实验中发现, 陶 粒对 TP 的吸附效果远优于页岩和沸石, 陶粒对磷的最 大吸附量可达 $714.29 \mathrm{mg} / \mathrm{kg}$, 与蒋丽等 ${ }^{[23]}$ 的研究结果 相当.

沸石、页岩、陶粒都是常见的人工湿地基质, 张迎颖 等 ${ }^{[24]}$ 以静态吸附试验研究湿地填料净化磷的性能, 发 现单一种类填料对 TP 的去除效果不太理想, 通常需要 多级连用以达到较好的效果. 本研究利用沸石、页岩、陶 粒填料,采用 3 级串联的配置方式, 总体上达到了较高 的磷去除效率.

\section{3 小结}

1) 阶梯垂直流人工湿地对 COD 具有稳定的去除效 果, 未受到不同分级进水方式的影响, 大部分的 COD 在系统第 1 级去除, 各系统 3 级出水浓度均能达到《城 镇污水处理厂污染物排放标准》( GB 18918-2002) 的一级 A 标准. 
2) 阶梯垂直流人工湿地对 TP 去除效果明显,各级均具有一定的 TP 去除能力,其中第 3 级 TP 去除贡献 最为明显, 陶粒有利于污水除磷. 系统 TP 出水浓度基本能达到《城镇污水处理厂污染物排放标准》( GB 18918-2002) 的一级 B 标准, 可以通过适宜基质的配置进一步降低 TP 出水浓度.

3 ) 分级进水比例对系统的氮去除效果影响显著. 当该比例为 $20 \%$ 时, TN 去除效率最高, 达 $61.70 \%$, 质 量去除速率为 $2.28 \mathrm{~g} /\left(\mathrm{m}^{2} \cdot \mathrm{d}\right)$. 该结果可以为阶梯垂直流人工湿地的设计和运行提供依据. 分级进水能有 效提高氮的去除效率, 后续可通过研究反硝化速率以及功能基因丰度等阐明分级进水对系统脱氮的影响及 其机制.

\section{4 参考文献}

[ 1 ] Perfler R, Laber J, Langergraber G et al. Constructed wetlands for rehabilitation and reuse of surface waters in tropical and subtropical areas-First results from small-scale plots using vertical flow beds. Water Science and Technology, 1999, 40 (3) : 155-162.

[ 2 ] Cheng S, Grosse W, Karrenbrock F et al. Efficiency of constructed wetlands in decontamination of water polluted by heavy metals. Ecological Engineering, 2002, 18(3): 317-325.

[ 3 ] Vymazal J, Kröpfelová L. Multistage hybrid constructed wetland for enhanced removal of nitrogen. Ecological Engineering, 2015, 84: 202-208.

[ 4 ] Zhang CC, Yin Q, Wen Y et al. Enhanced nitrate removal in self-supplying carbon source constructed wetlands treating secondary effluent: The roles of plants and plant fermentation broth. Ecological Engineering, 2016, 91: 310-316.

[ 5 ] Sikora FJ, Tong Z, Behrends LL et al. Ammonium removal in constructed wetlands with recirculating subsurface flow: Removal rates and mechanisms. Water Science and Technology, 1995, 32(3) : 193-202.

[ 6 ] Vymazal J. Horizontal sub-surface flow and hybrid constructed wetlands systems for wastewater treatment. Ecological Engineering, 2005, 25(5): 478-490.

[ 7 ] Vymazal J. Removal of nutrients in various types of constructed wetlands. Science of the Total Environment, 2007, 380(13SI ) : 48-65.

[ 8 ] Wu HM, Zhang J, Ngo HH et al. A review on the sustainability of constructed wetlands for wastewater treatment: Design and operation. Bioresource Technology, 2015, 175: 594-601.

[ 9 ] Tang Mengxuan, Wu Juan, Dai Yanran et al. Treatment of domestic wastewater by hybrid vertical constructed wetland. Chinese Journal of Environmental Engineering, 2016, (3) : 1017-1022. [唐孟煊, 吴娟, 代嫣然等. 组合式垂直流人工 湿地工艺及其污水处理效果. 环境工程学报, 2016, (3) : 1017-1022.]

[10] Rice EW. Standard methods for examination of water and wastewater 2012. Amer Public Health Assn, 2012.

[11] Xie Long, Wang Deguan, Dai Yu. Modelling organic matter removal in horizontal subsurface consstructed wetland. Chinese Environmental Science, 2009, (5) : 502-505. [谢龙, 汗德爟, 戴昱. 水平潜流人工湿地有机物去除模型研究. 中国 环境科学, 2009, (5): 502-505.]

[12] Vymazal J, Brix H, Cooper PF et al eds. Removal mechanisms and types of constructed wetlands. Backhuys Publisher, 1998: 17-66.

[13] Cooper PF, Job GD, Green MB et al. Reed beds and constructed wetlands for wastewater treatment. Medmenham, Marlow, UK: WRc Publicatiobs, 1996: 184.

[14] Li Jianbo. Wastewater treatment using enhanced hybrid constructed wetland system of vertical-flow and horizontal-flow [Dissertation]. Shanghai: Tongji University, 2008. [李剑波. 强化垂直流-水平流组合人工湿地处理生活污水研究 [学位论文].上海: 同济大学, 2008.]

[15] Li F, Lu L, Zheng X et al. Enhanced nitrogen removal in constructed wetlands: effects of dissolved oxygen and step-feeding. Bioresource Technology, 2014, 169(5) : 395-402.

[16] Kuenen JG. Anammox bacteria: from discovery to applicatio. Nature Reviews Microbiology, 2008, 6(4) : 320-326.

[17] Fan J, Zhang B, Zhang J et al. Intermittent aeration strategy to enhance organics and nitrogen removal in subsurface flow constructed wetlands. Bioresource Technology, 2013, 141(7) : 117-122.

[18] Zhao YJ, Liu B, Zhang WG et al. Performance of pilot-scale vertical-flow constructed wetlands in responding to variation in influent $\mathrm{C} / \mathrm{N}$ ratios of simulated urban sewage. Bioresource Technology, 2010, 101(6) : 1693-1700. 
[19] Pant HK, Reddy KR, Lemon E. Phosphorus retention capacity of root bed media of sub-surface flow constructed wetlands. Ecological Engineering, 2001, 17(4) : 345-355.

[20] Reddy KR, Kadlec RH, Flaig E et al. Phosphorus retention in streams and wetlands: A review. Critical Reviews in Environmental Science and Technology, 1999, 29(1): 83-146.

[21] Kingston FJ, Posner AM, Quirk JP. Anion adsorption by goethite and gibbsite: I. The role of the proton in determining adsorption envelopes. European Journal of Soil Science, 2010, 23(2) : 177-192.

[22] Lundager Madsen HE, Christensson F, Polyak LE et al. Calcium phosphate crystallization under terrestrial and microgravity conditions. Journal of Crystal Growth, 1995, 152(3) : 191-202.

[23] Jiang Li, Chen Jianyu, Li Xiaoming et al. Adsorption of phosphate from wastewater by fly ash ceramsite. Acta Scientiae Circumstantiae, 2011 , 31(7) : 1413-1420. DOI: 10.13671/j. hjkxxb. 2011.07.015. [蒋丽, 谌建宇, 李小明等. 粉煤灰陶 粒对废水中磷酸盐的吸附试验研究. 环境科学学报, 2011, 31(7) : 1413-1420.]

[24] Zhang Yingying, Ding Weimin, Chen Hao et al. Study on static adsorption characteristics and removal phosphorus of bulking agent. Jiangsu Agricultural Sciences, 2009, (3) : 416-418. [张迎颖, 丁为民, 陈豪等. 人工湿地填料的静态吸附 特性和动态除磷能力研究. 江苏农业科学, 2009, (3) : 416-418.] 